\title{
Dimethyl-2-oxoglutarate improves redox balance and mitochondrial function in muscle pericytes of individuals with diabetes mellitus
}

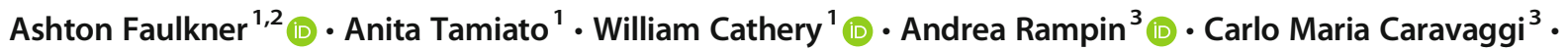 \\ Eva Jover ${ }^{1} \cdot$ Steve Allen $^{4} \cdot$ Harry Mellor $^{2}$ (D) $\cdot$ David Hauton $^{5} \cdot$ Lisa C. Heather $^{6} \cdot$ Gaia Spinetti $^{3}$ (D) $\cdot$ Paolo Madeddu $^{1}$
}

Received: 4 April 2020 / Accepted: 22 May 2020 / Published online: 30 July 2020

(C) The Author(s) 2020

\begin{abstract}
Aims/hypothesis Treatment of vascular complications of diabetes remains inadequate. We reported that muscle pericytes (MPs) from limb muscles of vascular patients with diabetes mellitus display elevated levels of oxidative stress causing a dysfunctional phenotype. Here, we investigated whether treatment with dimethyl-2-oxoglutarate (DM-2OG), a tricarboxylic acid cycle metabolite with antioxidant properties, can restore a healthy metabolic and functional phenotype.

Methods MPs were isolated from limb muscles of diabetes patients with vascular disease (D-MPs) and from non-diabetic control participants (ND-MPs). Metabolic status was assessed in untreated and DM-2OG-treated (1 mmol/l) cells using an extracellular flux analyser and anion-exchange chromatography-mass spectrometry (IC-MS/MS). Redox status was measured using commercial kits and IC-MS/MS, with antioxidant and metabolic enzyme expression assessed by quantitative RT-PCR and western blotting. Myogenic differentiation and proliferation and pericyte-endothelial interaction were assessed as functional readouts.

Results D-MPs showed mitochondrial dysfunction, suppressed glycolytic activity and reduced reactive oxygen speciesbuffering capacity, but no suppression of antioxidant systems when compared with ND-MP controls. DM-2OG supplementation improved redox balance and mitochondrial function, without affecting glycolysis or antioxidant systems. Nonetheless, this was not enough for treated D-MPs to regain the level of proliferation and myogenic differentiation of ND-MPs. Interestingly, DM-2OG exerted a positive effect on pericyte-endothelial cell interaction in the co-culture angiogenesis assay, independent of the diabetic status.

Conclusions/interpretation These novel findings support the concept of using DM-2OG supplementation to improve pericyte redox balance and mitochondrial function, while concurrently allowing for enhanced pericyte-endothelial crosstalk. Such effects may help to prevent or slow down vasculopathy in skeletal muscles of people with diabetes.
\end{abstract}

Keywords 2-Oxoglutarate $\cdot$ Diabetes mellitus $\cdot$ Mitochondria $\cdot$ Pericytes $\cdot$ Redox $\cdot$ Vascular protection

Electronic supplementary material The online version of this article (https://doi.org/10.1007/s00125-020-05230-4) contains peer-reviewed but unedited supplementary material, which is available to authorised users.

Paolo Madeddu mdprm@bristol.ac.uk

Ashton Faulkner ashton.faulkner@bristol.ac.uk

1 Bristol Medical School, Translational Health Sciences, University of Bristol, Upper Maudlin Street, Bristol BS2 8HW, UK

2 School of Biochemistry, University of Bristol, University Walk, Bristol BS8 1TD, UK
3 IRCCS, MultiMedica, Milan, Italy

4 Department of Comparative Biomedical Sciences, Royal Veterinary College, London, UK

5 Department of Chemistry, University of Oxford, Oxford, UK

6 Department of Physiology, Anatomy \& Genetics, University of Oxford, Oxford, UK 


\section{Research in context}

\section{What is already known about this subject?}

- Efforts to treat vascular complications of diabetes remain inadequate

- Skeletal muscle pericytes (MPs) represent an important angio-myogenic cell type that acts to support vascular networks, facilitate angiogenesis and maintain overall muscle function

- We previously reported that MPs from diabetes mellitus patients with vascular disease display elevated levels of oxidative stress, causing a dysfunctional phenotype

What is the key question?

- Can treatment of diabetic MPs (D-MPs) with dimethyl-2-oxoglutarate (DM-2OG), a tricarboxylic acid cycle metabolite with antioxidant properties, restore a healthy metabolic and functional phenotype?

\section{What are the new findings?}

- D-MPs showed mitochondrial dysfunction and reduced reactive oxygen species-buffering capacity, but no suppression of antioxidant systems when compared with non-diabetic MPs (ND-MPs)

- DM-2OG supplementation improved D-MP redox balance and mitochondrial function

- DM-2OG exerted a positive effect on pericyte-endothelial cell interaction in the co-culture angiogenesis assay but did not restore D-MP proliferation or myogenic differentiation to the levels measured in ND-MPs

How might this impact on clinical practice in the foreseeable future?

- These novel findings support the concept of using DM-2OG supplementation as a means of enhancing pericyteendothelial crosstalk, which may help to prevent or slow down vasculopathy in the skeletal muscle of people with diabetes

$\begin{array}{ll}\text { Abbreviations } \\ \text { CLI } & \text { Critical limb ischaemia } \\ \text { D-MP } & \text { Muscle pericyte from diabetic participant } \\ \text { DM-2OG } & \text { Dimethyl-2-oxoglutarate } \\ \text { EC } & \text { Endothelial cell } \\ \text { ECAR } & \text { Extracellular acidification rate } \\ \text { EGM2 } & \text { Endothelial cell growth medium 2 } \\ \text { GSH } & \text { Glutathione } \\ \text { GSSG } & \text { Glutathione (oxidised) } \\ \text { HIF1 } \alpha & \text { Hypoxia-inducible factor 1 } \alpha \\ \text { HO-1 } & \text { Haemoxygenase-1 } \\ \text { IC-MS/MS } & \text { Anion-exchange } \\ & \text { chromatography-mass-spectrometry } \\ \text { LDHA } & \text { Lactate dehydrogenase A } \\ \text { MEM } & \text { Minimum essential media } \\ \text { MP } & \text { Muscle pericyte } \\ \text { MSC } & \text { Mesenchymal stromal cell } \\ \text { MyHC } & \text { Myosin heavy chain } \\ \text { ND-MP } & \text { Muscle pericyte from non-diabetic participant } \\ \text { NHDF } & \text { Adult normal human dermal fibroblast } \\ \text { OCR } & \text { Oxygen consumption rate } \\ \text { 8-OHdG } & \text { 8-Hydroxy-2'-deoxyguanosine } \\ \text { 2OG } & \text { 2-Oxoglutarate } \\ \text { PECAM } & \text { Platelet endothelial cell adhesion molecule } \\ \text { PFA } & \text { Paraformaldehyde } \\ \text { PKC } \beta I I ~ & \text { Protein kinase C, isoform } \beta \text { II }\end{array}$

$\begin{array}{ll}\text { ROS } & \text { Reactive oxygen species } \\ \text { RT-qPCR } & \text { Quantitative RT-PCR } \\ \text { TCA } & \text { Tricarboxylic acid }\end{array}$

\section{Introduction}

Globally, an estimated 463 million people are currently living with diabetes mellitus [1], a major risk factor for cardiovascular disease $[2,3]$. This number is projected to rise over the next decade [1]. Muscle pericytes (MPs) represent an important angio-myogenic cell type that interact with endothelial cells (ECs) to support vascular networks and facilitate angiogenesis $[4,5]$. Diabetes-driven disruption in pericyte-EC interaction is an important cause of vessel instability and ischaemia [6, 7]. The mechanisms involved are not fully understood but alterations in metabolic flexibility and induction of oxidative stress is thought to play a role $[6,8]$. In line with this, we reported a reduction in number and function of supporting MP-like cells of diabetes patients presenting with critical limb ischaemia (CLI) [9]. Furthermore, these diabetic MPs (D-MPs) had elevated levels of reactive oxygen species (ROS) and exerted adverse effects on ECs.

Current treatments to reverse diabetic vascular complications remain sub-optimal, with approaches targeting metabolic and oxidative stress continuing to be actively pursued [8]. 
Given that the diabetic metabolic milieu can imprint on vascular cells, causing long-lasting changes beyond the restoration of systemic glucose control [10], a therapeutic option that sits at the metabolic/signalling interface may have significant impact. Metabolic intermediates of the tricarboxylic acid (TCA) cycle may represent one such interface. Among these, 2-oxoglutarate (2OG) may be a promising candidate.

In addition to its role in TCA cycle metabolism, 2OG has been shown to exert a protective effect through its ability to increase availability and activity of enzymatic and nonenzymatic antioxidant systems [11-14]. In this capacity, $2 \mathrm{OG}$ appears to have beneficial effects in models of ischaemic and oxidative stress [11,13, 15-17]. Furthermore, supplementation with $2 \mathrm{OG}$ has been found to extend cellular lifespan by decreasing mitochondrial oxidative phosphorylation, indicating a possible beneficial effect on the ageing process [18]. More recently, 2OG has been suggested to improve the functional activity of cardiac mesenchymal stromal cells (MSCs) isolated from diabetic participants [19]. This effect was mediated, at least in part, through reducing the hyper-methylated state and improving glucose uptake and mitochondrial function, suggesting that this metabolite might prove beneficial as an additional therapy in diabetic individuals with cardiovascular disease. However, whether $20 \mathrm{O}$ can exert beneficial effects on diabetes-induced pericyte dysfunction has not been investigated.

Given these perceived beneficial effects, the aim of this study was to characterise the metabolic and functional changes in pericyte-like cells isolated from diabetic participants presenting with CLI, and to investigate whether dimethyl$2 \mathrm{OG}$ (DM-2OG), a cell permeable $2 \mathrm{OG}$ derivative, could act as a 'therapeutic metabolite' to restore a healthy metabolic and functional phenotype.

\section{Methods}

\section{Cell culture}

Human samples were obtained with written informed consent. Their use conformed to the principles outlined in the Declaration of Helsinki and approved by the MultiMedica Research Ethics Committee (protocol \#011/2009). MPs were isolated from: (1) different anatomical locations of the lower extremities from non-diabetic control participants, referred for investigations/therapeutic interventions related to leg varicosity; or (2) foot muscle from type 1 and type 2 diabetic participants at the occasion of minor amputation for CLI (diagnosed according to the Trans-Atlantic Inter-Society Consensus Document on Management of Peripheral Arterial Disease [TASC] 2007 guidelines). Characteristics of the study population can be found in electronic supplementary material (ESM) Table 1.
MP isolation was performed following a well-established procedure described previously $[9,20]$ (see ESM method: Muscle pericyte isolation). MPs were sub-cultured in $\alpha$ minimum essential media (MEM) (20\% vol./vol. FBS) and used for experimentation between passages 2 and 5. For all experiments, no randomisation protocol was applied and investigators were not blinded to the group assignment or to the experimental results.

HUVECs were purchased from Lonza (Slough, UK) and cultured on $1 \%$ gelatine in endothelial cell growth medium 2 (EGM2). Adult normal human dermal fibroblasts (NHDFs) were purchased from Promocell (Heidelberg, Germany) and expanded in fibroblast growth medium before being transferred to EGM2 for experimentation.

\section{PKC $\beta$ II overexpression}

HEK293-T cells expanded in DMEM (10\% vol./vol. FBS) were transfected with a pLVX-puro plasmid (Clontech, UK) containing the human protein kinase $\mathrm{C}$, isoform $\beta$ II (PKC $\beta$ II) sequence under the control of a cytomegalovirus (CMV) promoter. pLVX-puro-GFP was used as a control. Cells were collected $48 \mathrm{~h}$ post transfection and processed for RNA and protein analysis.

\section{Cell viability}

MP viability was assessed using the Promega (Southampton, UK) LDH-glo Viability assay following the manufacturer's instructions (see ESM method: Cell viability).

\section{Functional analysis}

Angiogenesis Angiogenesis was assessed using the $3 \mathrm{D}$ coculture angiogenesis assay as previously described [21, 22]. NHDFs were cultured to confluence in EGM2 in 24-well plates. MPs and HUVECs (1:4 ratio) were seeded in EGM2 directly on top of the confluent NHDFs (technical duplicate). MPs were pre-stained with DiI (1,1'-dioctadecyl-3,3,3',3'tetramethylindocarbocyanine perchlorate; $1: 200 ; 20 \mathrm{~min}$ ). Following incubation for 7 days (medium and treatment refreshed every $48 \mathrm{~h}$ ), cells were fixed using 4\% (wt/vol.) paraformaldehyde (PFA), and vessel structures were viewed by staining with anti-platelet endothelial cell adhesion molecule (PECAM) antibody (R\&D systems BBA7; 1:200). Images were acquired using a Zeiss Axio Observer.Z1 fluorescence microscope (Carl Zeiss, UK) and analysed manually using ImageJ (v 1.52) software (https://imagej.nih.gov/ij/ index.html).

Proliferation MPs were seeded in 96-well plates (1000 cells/ well in triplicate) in the absence or presence of $1 \mathrm{mmol} / 1 \mathrm{DM}$ 2OG (Sigma Aldrich, UK). Proliferation over $72 \mathrm{~h}$ was 
quantified using a BrdU incorporation kit (Sigma Aldrich) following the manufacturer's instructions. Medium and treatment were refreshed every $24 \mathrm{~h}$. BrdU $(10 \mu \mathrm{mol} / \mathrm{l})$ was added for the final $24 \mathrm{~h}$ (see ESM method: Proliferation).

Myogenic differentiation MPs were seeded in 8-well chamber slides and cultured at confluence for 7 days to allow cell fusion and myotube formation. Cells were fixed with $4 \%$ (wt/vol.) PFA and permeabilised with $0.3 \%$ (vol./vol.) Triton X-100. Myogenic differentiation was assessed by probing cells overnight $\left(4^{\circ} \mathrm{C}\right)$ with anti-myosin heavy chain (MyHC) antibody (R\&D Systems MAB4470; clone MF20; 1:200). Nuclei were counter-stained using DAPI. Images were acquired using a Zeiss Axio Observer.Z1 fluorescence microscope and analysed using ImageJ software.

Permeability Permeability was assessed by measuring the transfer of FITC-dextran $(4 \mathrm{kDa}$ or $70 \mathrm{kDa})$ through HUVEC monolayers in transwell tissue culture plates (see ESM method: Permeability).

\section{RT-qPCR}

RNA was extracted using the Qiagen RNeasy Plus Mini Kit (Qiagen, UK) according to the manufacturer's instructions. Complementary DNA was synthesised using SuperScript II Reverse Transcriptase (ThermoFisher Scientific, UK). Quantitative RT-PCR (RT-qPCR), using intercalator (SYBR Premix Ex-Taq; TaKara Bio, France) and TaqMan technologies, was performed to determine changes in gene expression. PRKCB (Hs00176998_m1) and TBP (Hs00427620_m1) TaqMan primer-probes were obtained from ThermoFisher Scientific and SHC1 (H1_SHC1) was obtained from Sigma Aldrich. All other primer sequences were designed using Primer3 version 4.1.0 (http://primer3.ut.ee/) and NCBI primer-BLAST software (https://www.ncbi.nlm.nih.gov/ tools/primer-blast/) or obtained from published literature (ESM Table 2). Analysis was performed using the $2^{-\Delta \Delta C_{t}}$ method with results normalised to $T B P$ control gene.

\section{Western blotting}

Cells were lysed in RIPA buffer supplemented with protease/ phosphatase inhibitor cocktail (Sigma Aldrich). Protein concentration was determined by bicinchoninic acid (BCA) Protein Assay (ThermoFisher Scientific). Proteins $(25 \mu \mathrm{g})$ were separated by SDS-PAGE (60-90 $\mathrm{min} ; 120 \mathrm{~V})$. Resolved proteins were transferred to PVDF membranes (0.22 $\mu \mathrm{m}$; GE Healthcare, UK) by semi-dry transfer (90 min; $22 \mathrm{~V}$ ). Membranes were blocked (5\% wt/vol. skimmed (non-fat) milk; $1 \mathrm{~h}$ ) and probed with primary antibodies overnight $\left(4^{\circ} \mathrm{C}\right)(\mathrm{ESM}$ Table 3$)$ followed by incubation with ECL-HRP-conjugated secondary antibodies $(1: 10,000$; $1 \mathrm{~h}$ ) (GE Healthcare). Proteins were detected by enhanced chemiluminescence using Amersham ECL reagent (GE Healthcare). Band densities were semi-quantified by densitometry using Image $J$ and normalised to $\beta$-actin loading control.

\section{Immunocytochemistry}

Cells were fixed in $4 \%(\mathrm{wt} / \mathrm{vol}$.) PFA (10-15 min) and permeabilised in $0.1 \%$ (vol./vol.) Triton X-100 (15 min). Cells were incubated in blocking buffer $(5 \%$ vol./vol. FBS in PBS-Tween20; $45 \mathrm{~min})$ prior to overnight $\left(4^{\circ} \mathrm{C}\right)$ incubation with primary antibody at optimised concentrations (ESM Table 4). Primary antibodies were detected with AlexaFluor-488, -568 or -647 secondary antibodies (1:400 in PBS-Tween20) (ThermoFisher Scientific). Samples were incubated with DAPI $(1: 1000 ; 3 \mathrm{~min})$ and images were collected using a Zeiss Axio Observer.Z1 fluorescence microscope.

\section{Metabolic analysis}

Anion-exchange chromatography-mass-spectrometry Metabolites were measured using anion-exchange chromatography-mass-spectrometry (IC-MS/MS), similar to that described previously [23]. Compounds were identified with reference to accurate mass and retention time compared with authenticated standards run in-house. All data were normalised to total DNA for each sample and expressed as relative abundance. Absolute abundance of adenine nucleotides (ATP/ AMP/ADP) was calculated with reference to standard curves (see ESM method: Anion-exchange chromatography MassSpectroscopy).

Seahorse extracellular flux analysis Changes in oxygen consumption rate (OCR) and extracellular acidification rate (ECAR) were measured using the Seahorse XFp extracellular flux analyzer (Agilent, UK). Baseline readings were obtained, followed by sequential addition of oligomycin $(2 \mu \mathrm{mol} / \mathrm{l})$, carbonyl cyanide-4-(trifluoromethoxy)phenylhydrazone (FCCP; $2 \mu \mathrm{mol} / \mathrm{l})$ and rotenone/antimycin-A $(0.5 \mu \mathrm{mol} / \mathrm{l}$ each). Three readings ( $5 \mathrm{~min}$ intervals) were obtained after each pharmacological agent and values normalised to protein content (see ESM method: Seahorse extracellular flux analysis).

Extracellular lactate accumulation Medium lactate was measured using ABX-Pentra-C200 chemistry analyser (HORIBA, UK) with results normalised to protein content (see ESM method: Extracellular lactate accumulation). 


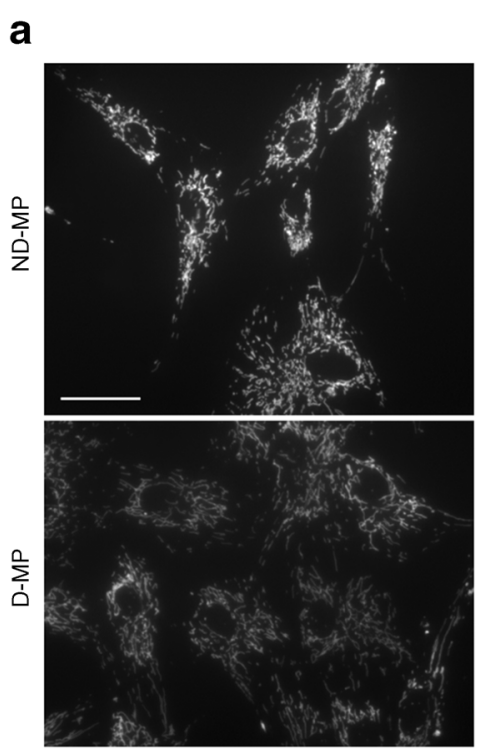

b
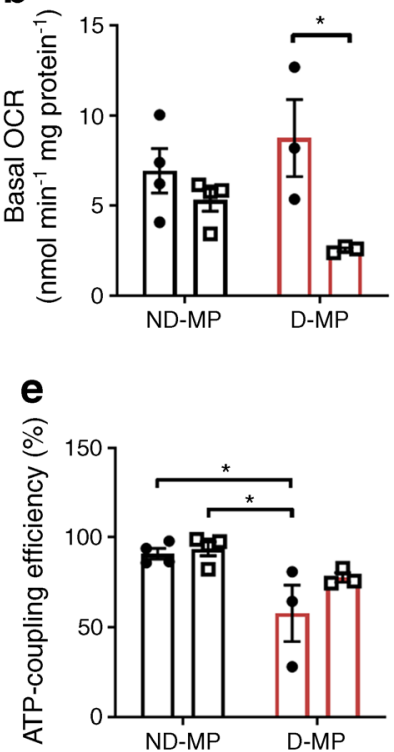

Fig. 1 (a) Representative images of MitoTracker-stained cells showing that gross mitochondrial morphology is not different between ND- and DMPs; scale bar, $20 \mu \mathrm{m}$. (b) Basal OCR is not different between untreated ND- and D-MPs but is significantly reduced in D-MPs supplemented with DM-2OG (1 mmol/l; $16 \mathrm{~h})$. (c) Maximal OCR and (d) spare capacity OCR are lower in D-MPs with no effect of DM-2OG supplementation. (e) ATP-coupling efficiency is significantly lower in D-MPs and is partially improved following DM-2OG supplementation. (f)

Measurement of $\mathrm{H}_{2} \mathrm{O}_{2}$ Cells (1000 cells/well 96-well plate) were incubated in the presence or absence of DM-2OG for $16 \mathrm{~h}$ (in triplicate). Medium $\mathrm{H}_{2} \mathrm{O}_{2}$ level was measured using the Promega ROS-Glo $\mathrm{H}_{2} \mathrm{O}_{2}$ Assay following the manufacturer's instructions (see ESM method: Measurement of $\mathrm{H}_{2} \mathrm{O}_{2}$ ).

Measurement of GSH/GSSG Cells were incubated for $16 \mathrm{~h}$ in the absence or presence of $1 \mathrm{mmol} / \mathrm{l} \mathrm{DM}-2 \mathrm{OG}$. Glutathione (GSH) was measured using the Promega GSH/GSSG-Glo Assay kit following the manufacturer's instructions. Results were normalised to protein content and the reduced GSH/ oxidised GSH (GSSG) ratio was calculated as GSH-GSSG/ (GSSG/2) (see ESM method: Measurement of GSH/GSSG).

\section{Mitochondrial assessment}

Mitochondrial DNA content Following extraction of DNA (QIAamp DNA Mini Kit; Qiagen, UK), a measure of mitochondrial to genomic DNA was performed by RT-qPCR using primers directed against mitochondrial-encoded tRNA-Leu(UUR) and nuclear-encoded B2-microglobulin, as described by Rooney et al [24].

Mitochondrial visualisation The mitochondrial-specific stain, MitoTracker Red-CMXRos (ThermoFisher Scientific), was used to visualise gross mitochondrial morphology by
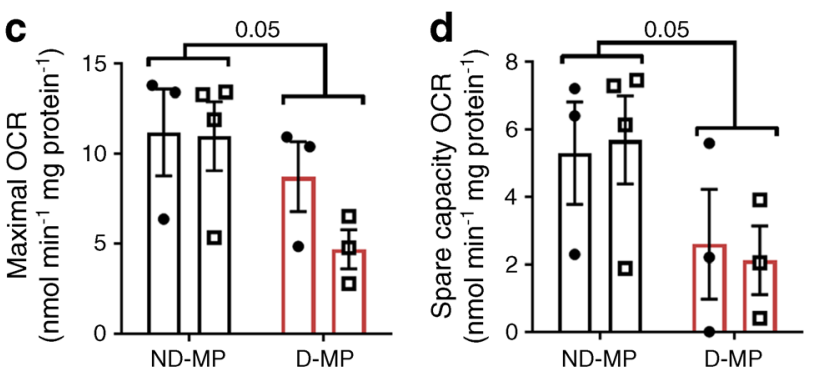

f
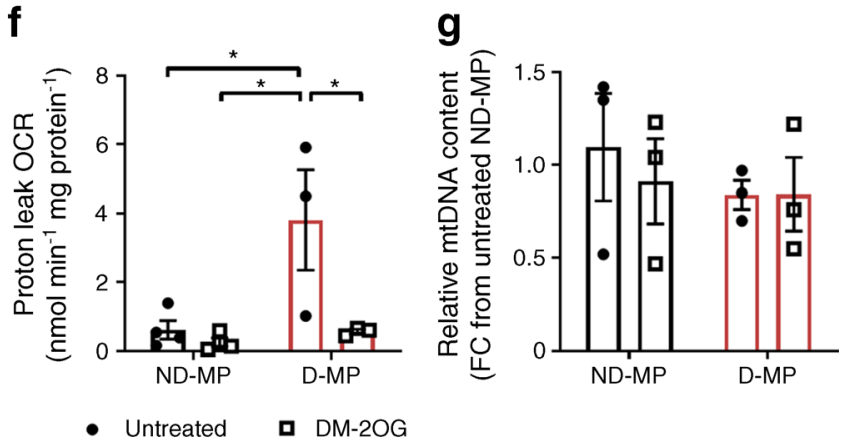

Mitochondrial proton leak is significantly higher in D-MPs and is restored towards the level of ND-MPs following supplementation with DM-2OG. (g) No change in relative mitochondrial DNA content was observed between ND- and D-MPs with or without DM-2OG treatment. Data are means ( \pm SEM) of $n=3-4$ for ND-MP and $n=3$ for D-MP; ${ }^{*} p<0.05$ as determined by two-way ANOVA followed by Tukey's post-comparison test. Numbers above bars in $(\mathbf{c}, \mathbf{d})$ indicate $p$ values. FC, fold change

fluorescence microscopy. Cells were incubated in $\alpha$-MEM containing $50 \mathrm{nmol} / 1$ MitoTracker Red-CMXRos dye (30 min). Images were acquired using a Zeiss Axio Observer.Z1 fluorescence microscope $(\times 40$ objective) .

\section{Statistical analysis}

Data are expressed as means \pm SEM from independent experiments performed on cell isolates from separate donors. Data analysis was performed using GraphPad Prism 6 software. Student's $t$ test was employed for establishing significant differences between two groups. For comparison of three or more groups, ANOVA was performed followed by an appropriate post hoc test. In all cases, $p<0.05$ indicated statistical significance.

\section{Results}

\section{Isolated cells display typical pericyte-related markers}

MP-like cells isolated from diabetic patients presenting with CLI (D-MPs), together with non-diabetic control participants (ND-MPs), were screened for a combination of pericyteassociated markers. In line with our previous report [9], isolated cells were confirmed to be positive for platelet-derived 


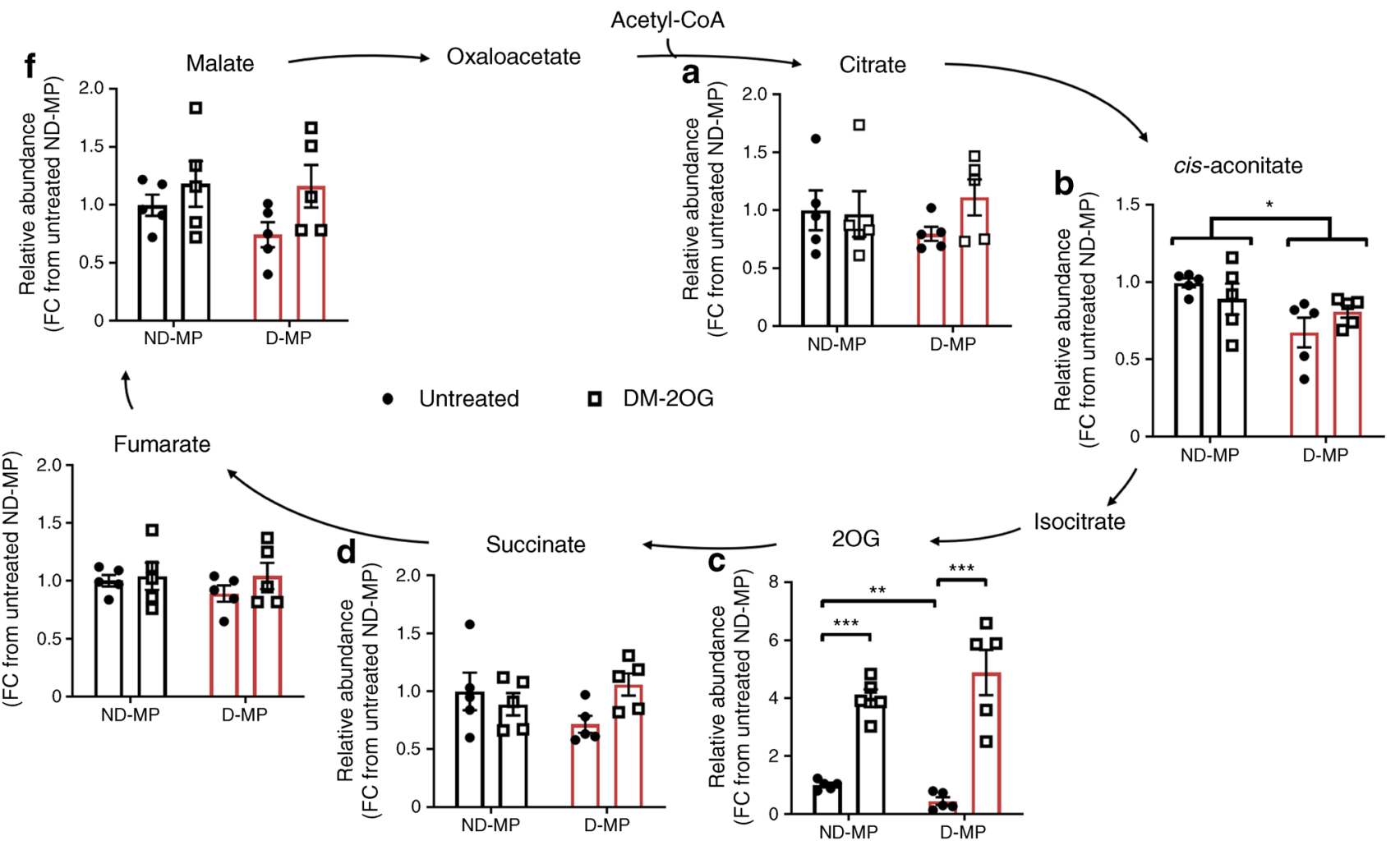

Fig. 2 ND- and D-MPs have similar levels of citrate (a), succinate (d), fumarate (e) and malate (f). D-MPs have significantly reduced levels of endogenous cis-aconitate (b) and 2OG (c) compared with ND-MPs. Supplementation with exogenous DM-2OG (1 mmol/l; $16 \mathrm{~h})$ significantly increased the intracellular abundance of $2 \mathrm{OG}$ in both

ND- and D-MPs without affecting other TCA cycle intermediates. Data represent means ( \pm SEM) of $n=5 ; * p<0.05, * * p<0.01$, $* * * p<0.001$ as determined by two-way ANOVA followed by Student's $t$ test. FC, fold change

growth factor receptor $\beta$ (PDGFR $\beta$ ), neuron-glial antigen (NG2) and CD146, and negative for endothelial marker CD31 (ESM Fig. 1). Expression of these markers did not differ between ND- and D-MPs (ESM Fig. 2a). Importantly, viability was similar between ND- and D-MP and was not affected by $1 \mathrm{mmol} / \mathrm{l}$ DM-2OG (ESM Fig. 2b), a concentration comparable with that of other studies [25-27].

\section{Mitochondrial function in D-MPs is improved by DM- 20G}

Mitochondrial network morphology was similar between NDand D-MPs (Fig. 1a), and no significant difference was observed in basal mitochondrial OCR (Fig. 1b). However, maximal OCR and spare capacity were reduced in D-MPs compared with ND-MPs (Fig. 1c, d). OCR coupled to ATP production was variable in D-MPs but when considered as a percentage of basal OCR (ATP-coupling efficiency), was significantly reduced compared with ND-MPs (Fig. 1e). Furthermore, D-MPs also showed a significant increase in non-ATP-linked OCR (mitochondrial proton leak) (Fig. 1f). Following supplementation with DM-2OG (16 h), basal OCR was reduced in D-MPs while maximal and spare capacity were largely unaffected. Moreover, the level of proton leak was significantly reduced back towards that measured in NDMPs. These changes were associated with a partial improvement in ATP-coupling efficiency, although ATP, ADP and AMP levels did not differ between ND- and D-MPs or following supplementation with DM-2OG (ESM Fig. 3). In addition, no effect of DM-2OG treatment was observed in ND-MPs (Fig. 1). Importantly, mitochondrial DNA content was similar between all treatment conditions (Fig. 1g), suggesting mitochondrial content remained unchanged.

To understand if changes in OCR were linked with alterations in TCA cycle activity, metabolite abundance was measured by IC-MS/MS. No difference was found in the level of citrate, succinate, fumarate or malate (Fig. 2). However, abundance of $c i s$-aconitate and $20 \mathrm{O}$ were significantly reduced in D-MPs. Following supplementation with exogenous DM-2OG, intracellular abundance of $2 \mathrm{OG}$ was significantly increased in both ND- and D-MPs, with no change in any other metabolite.

\section{Altered mitochondrial function is not a consequence of PKC $\beta$ II $/$ p66 $^{\text {SHC }}$ activation}

Upregulation of the PKC $\beta \mathrm{II} / \mathrm{p} 66^{\mathrm{SHC}}$ pathway has been reported as a prominent modulator of mitochondrial function and 

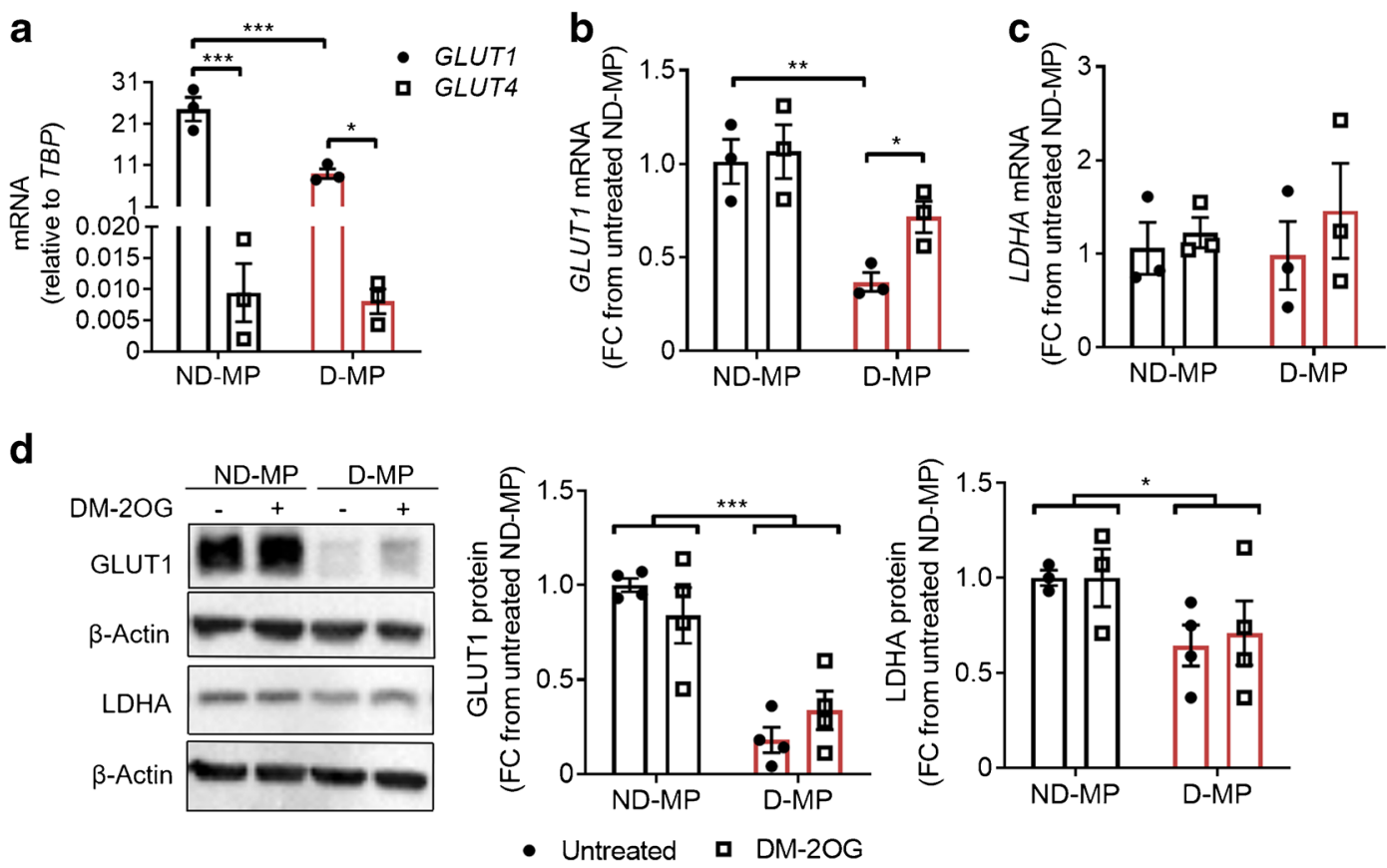

Fig. 3 (a) ND- and D-MPs have greater levels of GLUT1 compared with GLUT4 at the mRNA level. (b) D-MPs have a significantly reduced level of GLUT1 mRNA expression compared with ND-MPs. Supplementation with DM-2OG (1 mmol/1; $16 \mathrm{~h})$ significantly increases GLUT1 mRNA expression in D-MPs with no effect on ND-MPs. (c) LDHA mRNA expression is not significantly different between ND- and D-MPs with or without DM-20G supplementation. (d) Representative western blots

showing GLUT1, LDHA and $\beta$-actin loading control (see ESM Fig. 7 for original unedited blots) and associated densitometry analysis showing that D-MPs have a significantly reduced expression of GLUT1 and LDHA protein compared with ND-MPs, with no significant effect of DM-2OG supplementation. Data are means ( \pm SEM) of $n=3-4$; $* p<0.05, * * p<0.01, * * * p<0.001$ as determined by two-way ANOVA followed by Bonferroni's post-comparison test. FC, fold change

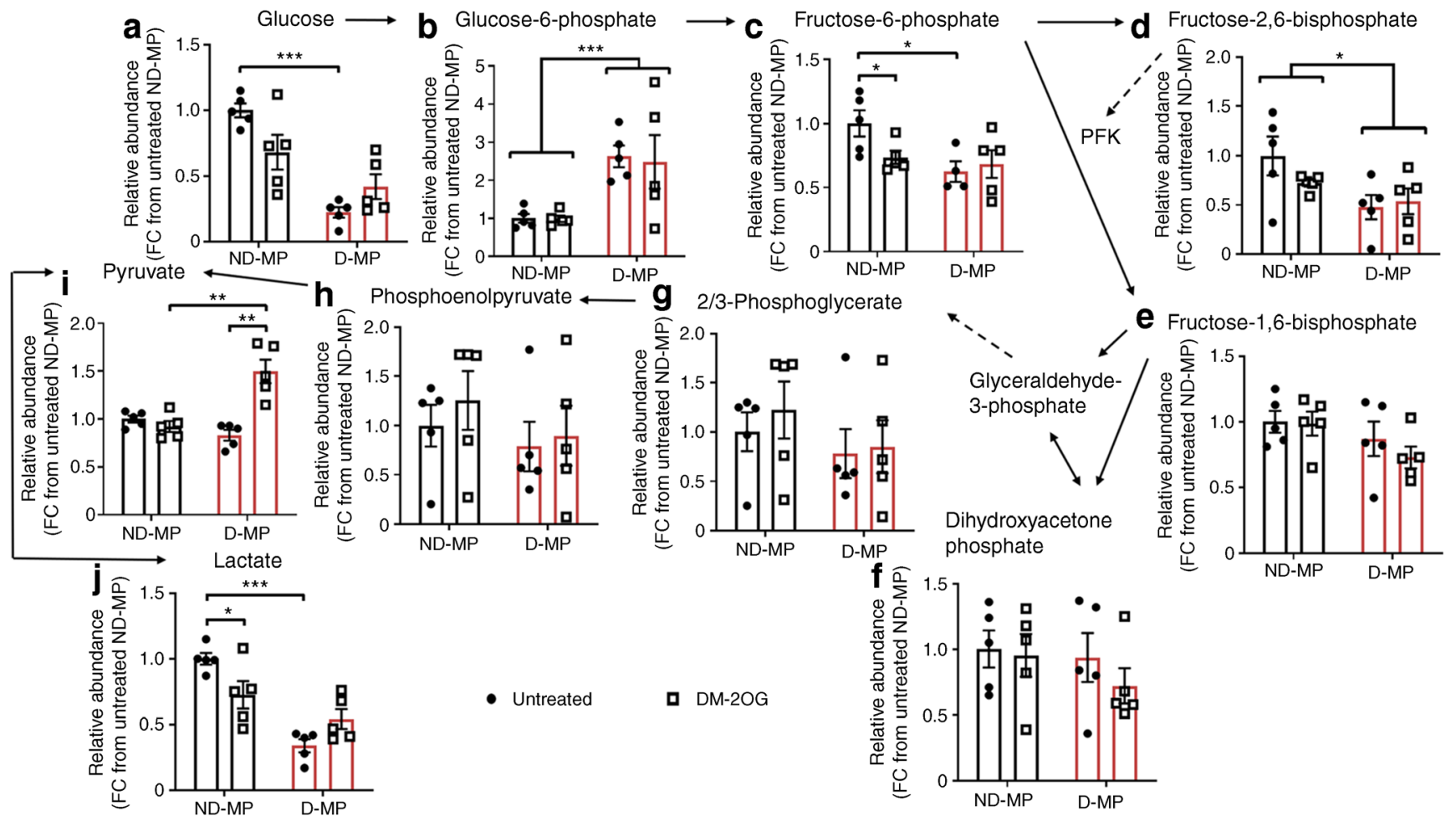

Fig. 4 Relative abundance of some glycolysis intermediates $(\mathbf{a}-\mathbf{j})$ is significantly altered in D-MPs compared with ND-MPs, indicating reduced glycolytic activity. DM-2OG supplementation $(1 \mathrm{mmol} / \mathrm{l} ; 16 \mathrm{~h})$ significantly reduces the abundance of fructose-6-phosphate (c) and

lactate (j) in ND-MPs but increases pyruvate abundance (i) in D-MPs. Data represent means $( \pm \mathrm{SEM})$ of $n=5 ; * p<0.05$, ** $p<0.01$, $* * * p<0.001$ as determined by two-way ANOVA followed by Student's $t$ test. FC, fold change; PFK, phosphofructokinase 
ROS production [28-30]. However, RT-qPCR and western blot analysis revealed that MPs did not express PKC $\beta I I$ to an extent that could be reliably detected (ESM Fig. 4). Adequate performance of primers and antibody against human PKC $\beta I I$ was confirmed using an overexpression system in HEK293-T cells. Furthermore, a significant reduction in the expression of the PKC $\beta I I$ target, $\mathrm{p} 66^{\mathrm{SHC}}$, was seen in D-MPs (ESM Fig. 4). These data suggest that this pathway is unlikely to be a significant contributing factor to the altered mitochondrial function observed in the D-MPs.

\section{Impaired glycolysis and metabolic flexibility in D-MPs is not rescued by DM-20G}

As glycolysis works alongside mitochondria to sustain vascular cell function [31, 32], and diabetes is known to affect this reciprocal interaction [33], we next investigated the impact of diabetes on markers of glycolysis and the effect of DM-2OG supplementation.

MPs expressed greater levels of the GLUT1 transporter compared with GLUT4 (Fig. 3a), and D-MPs had significantly less GLUT1 compared with ND-MPs at both mRNA and protein levels (Fig. 3b, d). D-MPs also had reduced lactate dehydrogenase A (LDHA) protein expression, but not mRNA, compared with ND-MPs (Fig. 3c, d). Following supplementation with DM-2OG $(16 \mathrm{~h})$, GLUT1 mRNA was restored in D-MPs, however, this was not observed at the protein level. Moreover, no effect of DM-2OG was observed on LDHA expression in D-MPs or on GLUT1 and LDHA expression in ND-MPs (Fig. 3b-d). Analysis of glycolytic intermediates revealed a significant reduction in glucose, fructose-6-phosphate, fructose-2,6-bisphosphate and lactate in D-MPs, and an accumulation in glucose 6-phosphate, further indicating supressed glycolytic activity (Fig. 4). Following supplementation with DM-2OG, ND-MPs had reduced abundance of fructose-6-phosphate and lactate, suggesting elevated $20 \mathrm{O}$ level reduces glycolytic activity (Fig. 4). In contrast, DM-2OG-treated D-MPs showed no alteration in metabolite abundance, except for an elevation in pyruvate, which may be the result of altered mitochondrial metabolism or change in other metabolic pathways involving this metabolite.

To understand if glycolytic capacity and metabolic flexibility were impacted, the ability to upregulate anaerobic glycolysis was investigated by measuring the change in lactate release under hypoxia $\left(2 \% \mathrm{O}_{2}\right)$. Compared with ND-MPs, D-MPs had reduced lactate release under normoxia and hypoxia, despite both significantly increasing mRNA expression of the hypoxia-inducible factor $1 \propto(\mathrm{HIF} 1 \alpha)$ target, GLUT1 (ESM Fig. 5a-c). Furthermore, while DM-2OG had no effect on lactate release by D-MPs, it was significantly reduced in ND-MPs under normoxic and hypoxic conditions (ESM Fig. 5a). However, the degree of upregulation following hypoxia was similar between untreated and DM-2OG-treated ND-MPs (ESM Fig. 5d). A similar change was observed when monitoring ECAR, a surrogate readout of lactate release, following pharmacological inhibition (oligomycin; $2 \mu \mathrm{mol} / \mathrm{l}$ ) of ATPsynthase activity (ESM Fig. 5e).

These data suggest that D-MPs have lower glycolytic activity and reduced metabolic flexibility, with less capacity for upregulating glycolysis when required. Supplementation with DM-2OG has little effect on D-MPs but lowers glycolytic activity in ND-MPs, without affecting their ability to respond to hypoxia.

\section{DM-20G has beneficial effects on D-MP redox status without upregulating antioxidant systems}

We previously demonstrated increased ROS burden in D-MPs [9], and given the changes in metabolic status described
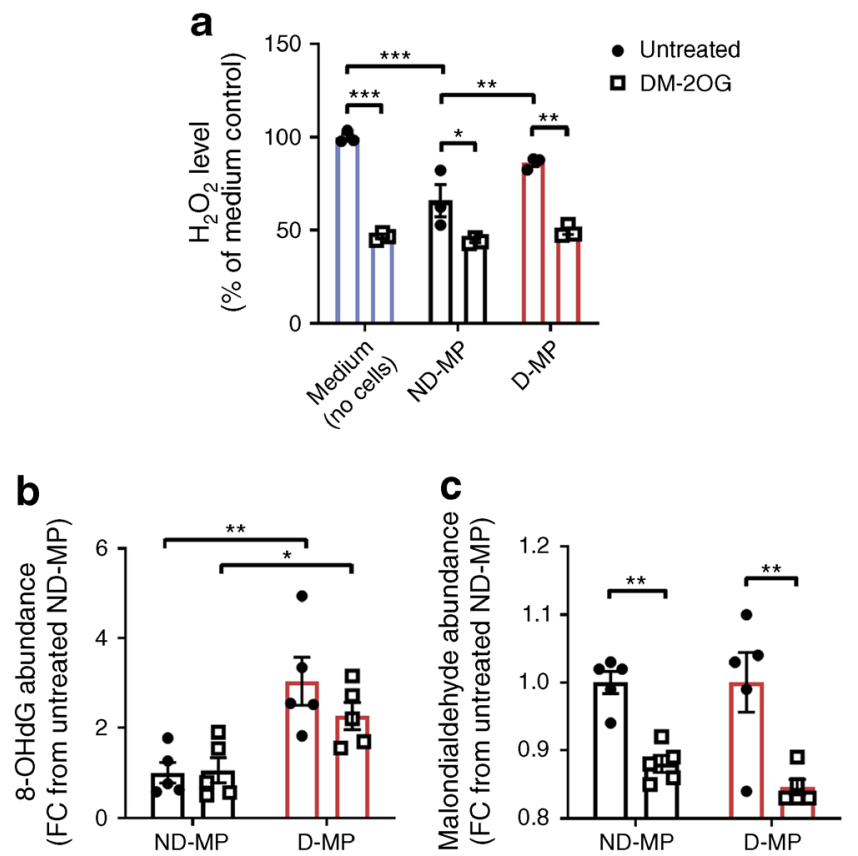

Fig. 5 (a) D-MPs have reduced $\mathrm{H}_{2} \mathrm{O}_{2}$ buffering activity compared with ND-MPs. Supplementation with DM-2OG (1 mmol/l; $16 \mathrm{~h})$ significantly reduces medium $\mathrm{H}_{2} \mathrm{O}_{2}$ level under all experimental conditions. Data represent mean $( \pm \mathrm{SEM})$ percentage of $\mathrm{H}_{2} \mathrm{O}_{2}$ level from untreated medium control; $n=3$; *p $<0.05$, ** $p<0.01$, *** $p<0.001$ as determined by two-way ANOVA followed by Bonferroni's post-comparison test. (b) DMPs have significantly greater levels of 8-OHdG, a marker of oxidative DNA damage, with levels unaffected by DM-2OG supplementation. (c) DM-2OG supplementation significantly reduces the relative abundance of malondialdehyde, a marker of lipid peroxidation, in both ND- and DMPs. For $(\mathbf{b}, \mathbf{c})$ data represent means $( \pm \mathrm{SEM})$ of $n=5 ; * p<0.05$, $* * p<0.01$ as determined by two-way ANOVA followed by Student's $t$ test. FC, fold change 
above, we further investigated the redox status in D-MPs and the ability of DM-2OG to restore redox balance.

D-MPs displayed reduced ability to buffer $\mathrm{H}_{2} \mathrm{O}_{2}$ within the culture medium compared with ND-MPs (Fig. 5a) and contained higher levels of 8-hydroxy-2'-deoxyguanosine (8OHdG), a marker of oxidative DNA damage (Fig. 5b). No difference was seen in the level of malondialdehyde, an indicator of lipid peroxidation (Fig. 5c). Supplementation with DM-2OG significantly reduced medium $\mathrm{H}_{2} \mathrm{O}_{2}$ under all experimental conditions (Fig. 5a). However, DM-2OG did not affect 8-OHdG level but significantly reduced malondialdehyde in both ND- and D-MPs (Fig. 5b, c).

To identify if the effects of DM-2OG were due to upregulating antioxidant defence, we assessed MP antioxidant status. NDand D-MPs had similar amounts of the $\mathrm{H}_{2} \mathrm{O}_{2}$-scavenging enzyme, catalase, at both the mRNA and protein level (Fig. 6a, c). In contrast, D-MPs had a small, but significant, increase in haemoxygenase-1 (HO-1) protein, a central antioxidant and cyto-protective enzyme, while mRNA levels remained unchanged (Fig. 6b, c). Furthermore, mRNA expression of the catalytic unit of a rate-governing enzyme of glutathione synthesis, glutamate-cysteine ligase (GCLC) (Fig. 6d), together with total GSH levels, as measured by IC-MS/MS (Fig. 6e) and the GSH/GSSG-Glo assay (Fig. 6f), were significantly increased in D-MPs compared with ND-MPs. Despite this upregulation, the reduced to oxidised GSH ratio (GSH/GSSG) was not significantly different (Fig. 6g). Importantly, none of these parameters were significantly altered by DM-2OG, suggesting that the beneficial effect of DM-2OG on ROS status is unlikely to be due to inducing antioxidant defence systems.

\section{Pericyte-endothelial interaction is enhanced by DM- 20G}

To understand whether the changes in metabolic and redox homeostasis are associated with altered functional activity, in vitro assessment of proliferation, myogenic differentiation and pericyte-endothelial interaction was performed.

D-MPs displayed a significantly reduced rate of proliferation compared with ND-MPs, and supplementation with DM$20 \mathrm{G}$ had a significant inhibitory effect on ND- but not D-MPs (Fig. 7a). Similarly, D-MPs showed less propensity to differentiate down the skeletal myocyte lineage, with no effect of DM-2OG being observed (Fig. 7b, c).

Given our previous identification of an adverse impact of DMPs on HUVEC 2D network formation [9], we further assessed the impact of MPs on EC angiogenic behaviour in a 3D coculture assay. The mean number and diameter of capillary-like tubes was not significantly different between wells containing ND- or D-MPs or following supplementation with DM-2OG
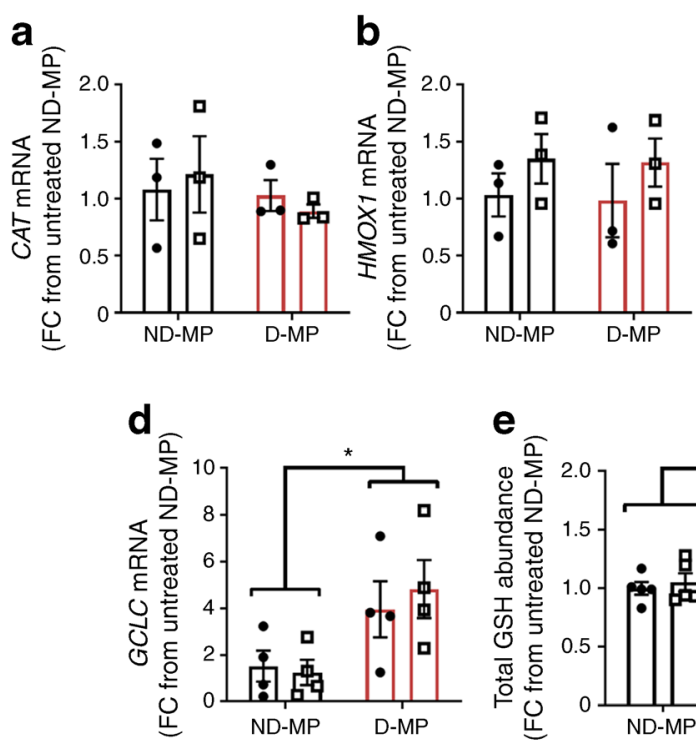

C
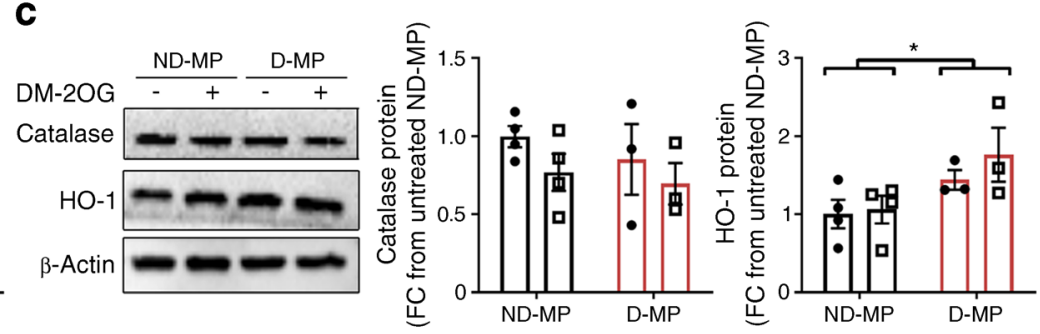

Fig. 6 (a) $C A T$ and (b) HMOX1 mRNA expression do not differ between ND- and D-MPs or following supplementation with DM-2OG (1 mmol/1; 16 h). Data represent means ( $\pm \mathrm{SEM}$ ) from $n=3$. (c) Representative western blot and associated densitometry analysis showing that HO-1 protein, but not catalase, is significantly increased in D-MPs with no effect of DM-2OG supplementation. Data represent means $( \pm$ SEM) from $n=4$ for ND-MP and $n=3$ for D-MP; $* p<0.05$ as determined by twoway ANOVA followed by Bonferroni's post-comparison test. See ESM Fig. 7 for original unedited blots. (d) GCLC mRNA expression is f

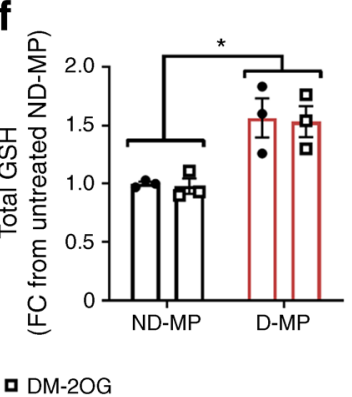

g

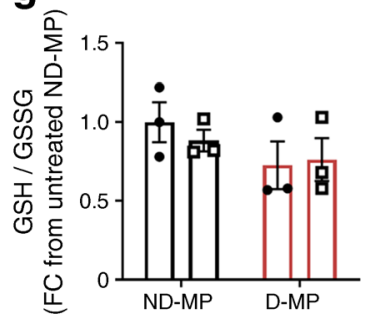

significantly higher in D-MPs, with no effect of DM-2OG supplementation. Data represent means ( $\pm \mathrm{SEM}$ ) from $n=4 ; * p<0.05$ as determined by two-way ANOVA followed by Bonferroni's post-comparison test. (e) IC-MS/MS $(n=5)$ and (f) GSH-Glo assay $(n=3)$ analysis showing that total GSH is significantly higher in D-MPs, with no effect of DM-2OG supplementation. (g) The GSH/GSSG ratio $(n=3)$ is not significantly different between ND- and D-MPs or following supplementation with DM-2OG. For $(\mathbf{e}-\mathbf{g})$ data represent means $( \pm \mathrm{SEM}) ; * p<0.05$, $* * p<0.01$ as determined by two-way ANOVA. FC, fold change 
(Fig. 8a, b). However, although MPs showed evidence of direct interaction with ECs, the mean distance between the MP and endothelial network was significantly greater with D-MPs (Fig. $8 \mathrm{c}, \mathrm{d}$ ), indicating reduced interaction/communication. This distance was significantly reduced in the presence of DM-2OG, an effect seen with both D- and ND-MPs, suggesting that DM$2 \mathrm{OG}$ can improve pericyte-endothelial interaction/crosstalk.

To assess whether improved interaction influences endothelial barrier properties, the effect of MPs and DM-2OG on HUVEC permeability was investigated. The presence of MPs significantly reduced the ability of large molecules (represented by $70 \mathrm{kDa}$ FITC-dextran) to pass through a HUVEC monolayer but had no effect on smaller molecules (represented by $4 \mathrm{kDa}$ FITC-dextran) (ESM Fig. 6). Moreover, no significant difference was seen between wells containing ND- or D-MPs, or those containing DM-2OG.

Together these data demonstrate that D-MPs have reduced proliferation and differentiation capacity and that DM-2OG supplementation is not able to rescue these deficiencies. DMPs do not adversely impact permeability or the angiogenic behaviour of healthy ECs but do display less interaction with the endothelial network, a deficit that is significantly improved by DM-2OG.

\section{Discussion}

The aim of the present study was to characterise the metabolic and functional changes in D-MPs and identify any beneficial effect of DM-2OG supplementation.

While the presence of D-MPs did not alter EC permeability or the ability of ECs to undergo tubulogenesis, D-MPs did demonstrate a reduction in their proliferative behaviour, reduced propensity to differentiate down the myogenic lineage, and reduced interaction with ECs. This impairment in function is not due to any significant change in cell viability, but instead can be explained, at least in part, through the observed changes in D-MP metabolic phenotype imparted by the diabetic milieu and probably compounded by the ischaemic environment from which the cells are isolated.

Cells utilise mitochondria alongside glycolysis to facilitate signalling and anabolic metabolism aimed at driving proliferation, migration and morphological re-arrangement [31, 32, 34]. We report that D-MPs have significant alterations in both glycolysis and mitochondrial metabolism that are in line with the observed decline in cell function. In agreement with earlier studies [35, 36], MPs predominantly expressed the GLUT1 transporter that, alongside LDHA, was significantly downregulated in D-MPs. Alongside this, D-MPs displayed reduced glycolytic activity and a lack of metabolic flexibility. Furthermore, D-MPs also presented with significant changes in mitochondrial function. Although mitochondrial network morphology and basal OCR was not significantly

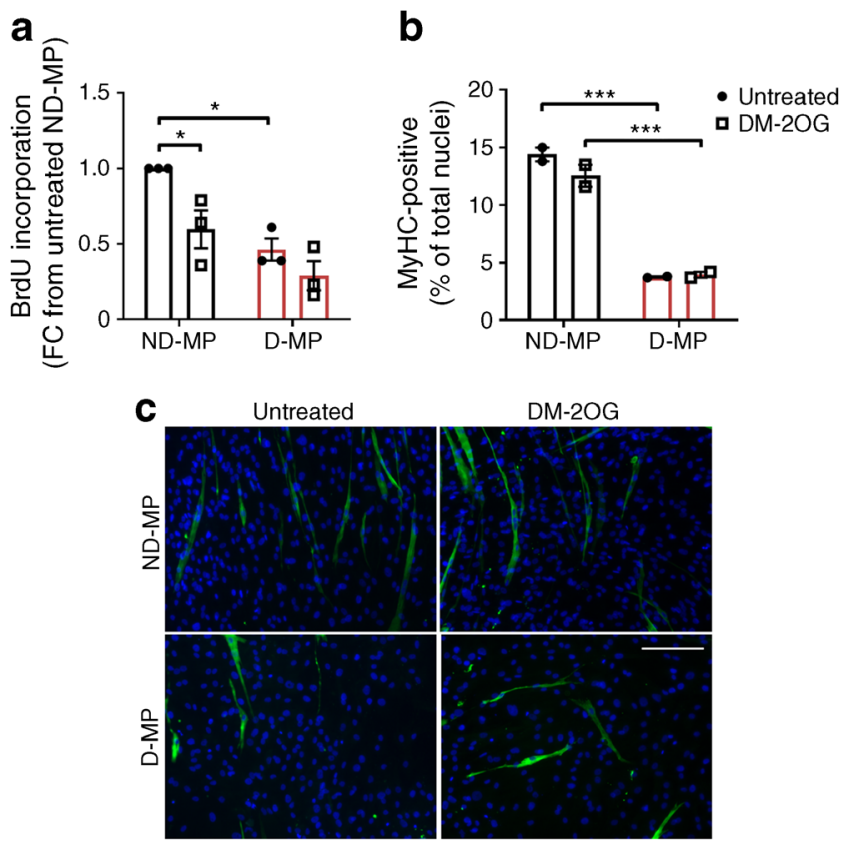

Fig. 7 (a) D-MPs show significantly reduced proliferation over $72 \mathrm{~h}$ compared with ND-MPs. Supplementation with DM-2OG $(1 \mathrm{mmol} / \mathrm{l}$; $72 \mathrm{~h}$ ) significantly reduces proliferation of ND- but not D-MPs. Data represent means $( \pm \mathrm{SEM})$ fold-change in BrdU incorporation from $n=3$; $* p<0.05$ as determined by two-way ANOVA followed by Tukey's postcomparison test. (b) Analysis and (c) representative immunofluorescence images (scale bar, $50 \mu \mathrm{m}$ ) showing that D-MPs have reduced propensity to differentiate down the myogenic lineage compared with ND-MPs at 7 days, with no effect of DM-2OG supplementation. Data represent means ( \pm SEM) percentage of nuclei associated with positive MyHC staining (green) from $n=2 ; * * * p<0.001$ as determined by two-way ANOVA followed by Bonferroni's post-comparison test. FC, fold change

compromised, D-MPs had reduced maximal and spare OCR capacities, together with reduced efficiency of oxygen-linked ATP production. D-MPs also showed an elevation in proton leak, an additional indicator of oxidative burden [37, 38]. Such blunted metabolic capacity would contribute to the reduced functional activity of D-MPs. Indeed, the importance of upregulating these metabolic networks for such behaviour has been demonstrated in other vascular cells [31, 32, 34].

Although it was not possible to identify a specific inducer of the mitochondrial dysfunction, it is possible to rule out activation of PKC $\beta I I$, as we were unable to detect this protein kinase. Unlike other cells, the role of the PKC $\beta \Pi / \mathrm{p} 66^{\mathrm{SHC}}$ pathway in pericytes is less studied and mainly restricted to the retina [39-41]. Even when detected, its role in pericyte biology and diabetes-related dysfunction remains unclear [41, 42].

Alterations in metabolism are often accompanied by changes in redox status/control that have a profound impact on vascular function [43]. We have shown that D-MPs have higher levels of oxidative stress [9], and in the present study, D-MPs displayed a greater level of oxidative damage, particularly towards DNA, and reduced capacity to buffer their environment. Importantly, this reduction in ROS-buffering does not appear to be due to compromised expression of antioxidant systems. 
Fig. 8 No significant difference was observed between wells containing ND- or D-MPs with regard to the mean number of branches (a) or mean branch diameter (b) formed by HUVECs in the 3D angiogenesis assay. (c) Quantification and (d) representative immunofluorescence images (scale bar, $50 \mu \mathrm{m}$ ) showing that the mean distance between pericytes (red; DiI) and HUVEC branches (green; PECAM) is significantly greater in wells containing D-MPs compared with ND-MPs and is significantly reduced when supplemented with DM-2OG (1 mmol/l; 7 days). Data represent means $( \pm$ SEM) from $n=3 ; * p<0.05, * * p<0.01$ as determined by two-way ANOVA followed by Bonferroni's post-comparison test

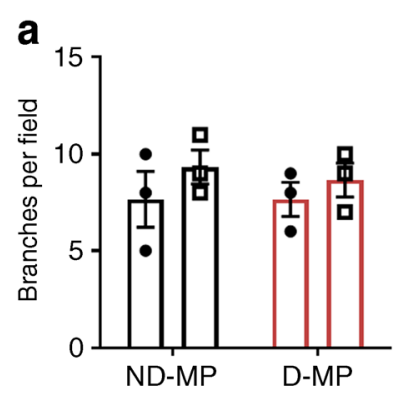

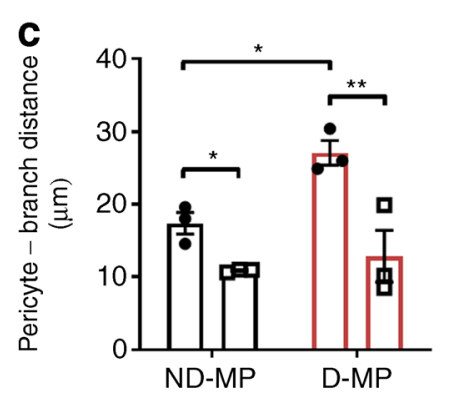

- Untreated a DM-2OG
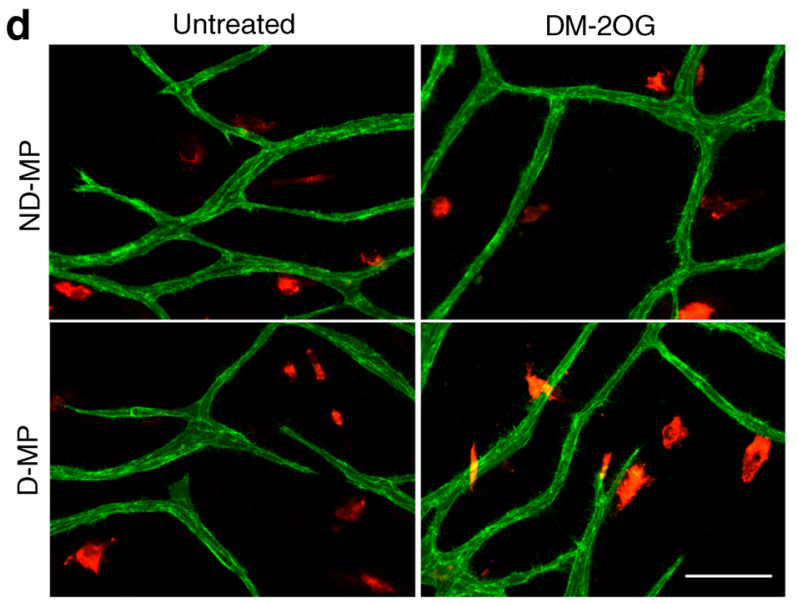

Given the potential role of $2 \mathrm{OG}$ as an antioxidant and signalling molecule [11-14, 44], we explored whether DM-2OG supplementation could exert a beneficial effect on metabolic balance to allow for restoration of D-MP function. This is particularly pertinent as endogenous $2 \mathrm{OG}$ abundance was reduced in D-MPs, suggesting that a lack of this metabolite may be a compounding factor in the observed functional decline.

Following DM-2OG supplementation, intracellular abundance of $20 \mathrm{O}$ was elevated. However, the lack of an effect on antioxidant systems contrasts with reports in other cell types $[12,14]$. Instead, it is possible that DM-2OG is acting, at least in part, as a direct scavenger of $\mathrm{H}_{2} \mathrm{O}_{2}$, as indicated by the reduction in $\mathrm{H}_{2} \mathrm{O}_{2}$ levels within the culture medium. Indeed, by virtue of its structure, direct $2 \mathrm{OG}$ interaction and clearance of $\mathrm{H}_{2} \mathrm{O}_{2}$ has previously been identified, presenting a complementary antioxidant mechanism [25, 45].

Reduction in oxidative stress is also consistent with the reduction in mitochondrial OCR and improved efficiency of oxygencoupled ATP production observed in DM-2OG-treated D-MPs, as lower OCR and more efficient mitochondria are less likely to produce excessive ROS [46]. A similar effect on oxygen utilisation has been reported previously, where interaction between $20 \mathrm{O}$ and mitochondrial ATP-synthase was described [18]. In the current study, DM-2OG supplementation also led to reduced OCR linked to proton leak, indicating reduced oxidative burden and improved mitochondrial function. The level of malondialdehyde was also lowered following DM-2OG supplementation, suggesting a potential protective effect on lipid peroxidation state, which could also benefit mitochondria. It is worth noting that apart from the reduction in malondialdehyde and medium $\mathrm{H}_{2} \mathrm{O}_{2}$ level, none of the above effects were evident in ND-MPs. This was not due to reduced DM-2OG uptake but is likely to be a consequence of its differential utilisation, with greater effects being imparted on reducing glucose metabolism. Although the reduction in glycolysis could be through $2 \mathrm{OG}$ acting as a positive co-factor for prolyl hydroxylase-mediated degradation of HIF $1 \alpha$, this does not appear to prevent the ability to respond to hypoxia or upregulate GLUT1 expression (a $\mathrm{HIF} 1 \alpha$ target) when required.

Given these differential metabolic effects, it is not surprising that DM-2OG was unable to restore D-MP proliferation and reduced proliferation of ND-MPs. The effect of $2 \mathrm{OG}$ on cell proliferation is variable within the literature [47-49], and our results contrast with those reported in cardiac MSCs, where $20 G$ was found to have no effect on metabolic or proliferative activity of non-diabetic MSCs, but rescued proliferation of diabetic MSCs in association with a rescue of glucose metabolism and mitochondrial function [19].

Importantly, DM-2OG was able to enhance pericyte-EC interaction in the angiogenesis assay. Although the mechanism needs identifying through further investigation, it is likely to involve a combination of effects imparted on both MPs and ECs. Interestingly, while investigating $2 \mathrm{OG}$ for preventing tumour progression in mice, greater pericyte 
coverage of vessels was observed following $2 \mathrm{OG}$ treatment [50]. When coupled with our own observation, this may indicate a potential role for $2 \mathrm{OG}$ in facilitating pericyteendothelial interaction and vessel stability, raising the possibility of utilising $2 \mathrm{OG}$ to prevent, or at least slow the rate of, vascular decline in individuals with diabetes.

These novel findings expand our understanding of the metabolic and behavioural dysfunction that occurs in the MPs of people with diabetes. Furthermore, these data support the concept of using DM-2OG as a means of improving pericyte redox balance and mitochondrial function, while simultaneously allowing for enhanced pericyte-endothelial interaction. Given the relatively low sample size assessed in this study, if these effects are shown to be representative of people with diabetes then the potential of DM-2OG to slow the rate of vascular decline in vivo warrants further investigation.

Acknowledgements The authors would like to acknowledge V. Ball (Department of Physiology, Anatomy \& Genetics, University of Oxford, UK) for assistance with measuring medium lactate levels.

Data availability The datasets generated during and/or analysed during the current study are available from the corresponding author on reasonable request.

Funding This work was supported by the British Heart Foundation (BHF) project grant 'Targeting of protein kinase $\mathrm{C}$ beta II (PKC $\beta I I)$ to improve vascular and muscular fitness in diabetic peripheral ischaemia' (PG/16/101/32622) awarded to PM. This work was also supported by grants from the Italian Ministry of Health, Ricerca Corrente to the IRCCS MultiMedica and from the Cariplo Foundation (rif\# 20160922). The funders were not involved in the design of the study; the collection, analysis and interpretation of data; writing of the report; and did not impose any restrictions regarding the publication of the report.

Authors' relationships and activities The authors declare that there are no relationships or activities that might bias, or be perceived to bias, their work.

Contribution statement AF conceived and designed the study, performed experiments, analysed and interpreted data and wrote the manuscript. AT, WC, AR, CMC, EJ, SA, HM, DH and LH contributed to acquisition, analysis and interpretation of data. GS conceived and designed the study, and analysed and interpreted data. PM conceived and designed the study, interpreted data and acquired funding. All authors revised the article for important intellectual content and approved the final version for publication. PM is the guarantor of this work.

Open Access This article is licensed under a Creative Commons Attribution 4.0 International License, which permits use, sharing, adaptation, distribution and reproduction in any medium or format, as long as you give appropriate credit to the original author(s) and the source, provide a link to the Creative Commons licence, and indicate if changes were made. The images or other third party material in this article are included in the article's Creative Commons licence, unless indicated otherwise in a credit line to the material. If material is not included in the article's Creative Commons licence and your intended use is not permitted by statutory regulation or exceeds the permitted use, you will need to obtain permission directly from the copyright holder. To view a copy of this licence, visit http://creativecommons.org/licenses/by/4.0/.

\section{References}

1. Saeedi P, Petersohn I, Salpea P et al (2019) Global and regional diabetes prevalence estimates for 2019 and projections for 2030 and 2045: Results from the International Diabetes Federation Diabetes Atlas, 9th edition. Diabetes Res Clin Pract 157:107843. https://doi. org/10.1016/j.diabres.2019.107843

2. Fowkes FGR, Rudan D, Rudan I et al (2013) Comparison of global estimates of prevalence and risk factors for peripheral artery disease in 2000 and 2010: a systematic review and analysis. Lancet 382(9901): 1329-1340. https://doi.org/10.1016/S0140-6736(13)61249-0

3. Spreen MI, Gremmels H, Teraa M et al (2016) Diabetes is associated with decreased limb survival in patients with critical limb ischemia: pooled data from two randomized controlled trials. Diabetes Care 39(11):2058-2064. https://doi.org/10.2337/dc16-0850

4. Cappellari O, Cossu G (2013) Pericytes in development and pathology of skeletal muscle. Circ Res 113(3):341-347. https://doi.org/ 10.1161/CIRCRESAHA.113.300203

5. Birbrair A, Zhang T, Wang Z-M, Messi ML, Mintz A, Delbono O (2014) Pericytes: multitasking cells in the regeneration of injured, diseased, and aged skeletal muscle. Front Aging Neurosci 6:245. https://doi.org/10.3389/fnagi.2014.00245

6. Warmke N, Griffin KJ, Cubbon RM (2016) Pericytes in diabetesassociated vascular disease. J Diabetes Complicat 30(8):16431650. https://doi.org/10.1016/j.jdiacomp.2016.08.005

7. Liu C, Ge H-M, Liu B-H et al (2019) Targeting pericyte-endothelial cell crosstalk by circular RNA-cPWWP2A inhibition aggravates diabetes-induced microvascular dysfunction. Proc Natl Acad Sci 116(15):7455-7464. https://doi.org/10.1073/pnas.1814874116

8. Teodoro JS, Nunes S, Rolo AP, Reis F, Palmeira CM (2019) Therapeutic options targeting oxidative stress, mitochondrial dysfunction and inflammation to hinder the progression of vascular complications of diabetes. Front Physiol 9:1857. https://doi.org/10. 3389/fphys.2018.01857

9. Vono R, Fuoco C, Testa S et al (2016) Activation of the pro-oxidant PKC 3 II-p66Shc signaling pathway contributes to pericyte dysfunction in skeletal muscles of patients with diabetes with critical limb ischemia. Diabetes 65(12):3691-3704. https://doi.org/10.2337/db16-0248

10. Luna P, Guarner V, Farías JM, Hernández-Pacheco G, Martínez M (2016) Importance of metabolic memory in the development of vascular complications in diabetic patients. J Cardiothorac Vasc Anesth 30(5):1369-1378. https://doi.org/10.1053/j.jvca.2016.02.008

11. Mariño G, Pietrocola F, Kong Y et al (2014) Dimethyl $\alpha$ ketoglutarate inhibits maladaptive autophagy in pressure overload-induced cardiomyopathy. Autophagy 10(5):930-932. https://doi.org/10.4161/auto.28235

12. He L, Wu J, Tang W et al (2018) Prevention of oxidative stress by $\alpha$-ketoglutarate via activation of car signaling and modulation of the expression of key antioxidant-associated targets in vivo and in vitro. J Agric Food Chem 66(43):11273-11283. https://doi.org/ 10.1021/acs.jafc. 8 b04470

13. Cheng M-X, Cao D, Chen Y, Li J-Z, Tu B, Gong J-P (2019) $\alpha$ Ketoglutarate attenuates ischemia-reperfusion injury of liver graft in rats. Biomed Pharmacother 111:1141-1146. https://doi.org/10. 1016/j.biopha.2018.12.149

14. Velvizhi S, Dakshayani KB, Subramanian P (2002) Effects of $\alpha$ ketoglutarate on antioxidants and lipid peroxidation products in rats treated with ammonium acetate. Nutrition 18(9):747-750. https:// doi.org/10.1016/S0899-9007(02)00825-0

15. Wang L, Hou Y, Yi D et al (2015) Dietary supplementation with glutamate precursor $\alpha$-ketoglutarate attenuates lipopolysaccharideinduced liver injury in young pigs. Amino Acids 47(7):1309-1318. https://doi.org/10.1007/s00726-015-1966-5

16. Wang L, Yi D, Hou Y et al (2016) Dietary supplementation with $\alpha$ ketoglutarate activates mTOR signaling and enhances energy status 
in skeletal muscle of lipopolysaccharide-challenged piglets. J Nutr 146(8):1514-1520. https://doi.org/10.3945/jn.116.236000

17. Tkachenko V, Kovalchuk Y, Bondarenko N et al (2018) The cardio- and neuroprotective effects of corvitin and 2-oxoglutarate in rats with pituitrin-isoproterenol-induced myocardial damage. Biochem Res Int 2018:2019302414

18. Chin RM, Fu X, Pai MY et al (2014) The metabolite $\alpha$ ketoglutarate extends lifespan by inhibiting ATP synthase and TOR. Nature 510:397-401. https://doi.org/10.1038/nature13264

19. Spallotta F, Cencioni C, Atlante S et al (2018) Stable oxidative cytosine modifications accumulate in cardiac mesenchymal cells from type 2 diabetes patients: rescue by $\alpha$-ketoglutarate and TETTDG functional reactivation. Circ Res 122(1):31-46. https://doi. org/10.1161/CIRCRESAHA.117.311300

20. Fuoco C, Sangalli E, Vono R et al (2014) 3D hydrogel environment rejuvenates aged pericytes for skeletal muscle tissue engineering. Front Physiol 5:203. https://doi.org/10.3389/fphys.2014.00203

21. Richards M, Hetheridge C, Mellor H (2015) The formin FMNL3 controls early apical specification in endothelial cells by regulating the polarized trafficking of podocalyxin. Curr Biol 25(17):23252331. https://doi.org/10.1016/j.cub.2015.07.045

22. Richards M, Mellor H (2016) In vitro coculture assays of angiogenesis. Methods Mol Biol 1430:159-166. https://doi.org/10.1007/ 978-1-4939-3628-1_10

23. Riffelmacher T, Clarke A, Richter FC et al (2017) Autophagydependent generation of free fatty acids is critical for normal neutrophil differentiation. Immunity 47(3):466-480. https://doi.org/10. 1016/j.immuni.2017.08.005

24. Rooney JP, Ryde IT, Sanders LH et al (2015) PCR based determination of mitochondrial DNA copy number in multiple species. Methods Mol Biol 1241:23-38. https://doi.org/10.1007/978-14939-1875-1_3

25. Long LH, Halliwell B (2011) Artefacts in cell culture: $\alpha$ ketoglutarate can scavenge hydrogen peroxide generated by ascorbate and epigallocatechin gallate in cell culture media. Biochem Biophys Res Commun 406(1):20-24. https://doi.org/10.1016/j. bbrc.2011.01.091

26. Yang Q, Liang X, Sun X et al (2016) AMPK/ $\alpha$-ketoglutarate axis dynamically mediates DNA demethylation in the Prdm16 promoter and brown adipogenesis. Cell Metab 24(4):542-554. https://doi. org/10.1016/j.cmet.2016.08.010

27. Zhao J, Peng L, Luo Z, Cui R, Yan M (2015) Inhibitory effects of dimethyl $\alpha$-ketoglutarate in hepatic stellate cell activation. Int J Clin Exp Pathol 8(5):5471-5477

28. Paneni F, Mocharla P, Akhmedov A et al (2012) Gene silencing of the mitochondrial adaptor p66Shc suppresses vascular hyperglycemic memory in diabetes. Circ Res 111(3):278-289. https://doi.org/ 10.1161/CIRCRESAHA.112.266593

29. Wojtala A, Karkucinska-Wieckowska A, Sardao VA et al (2017) Modulation of mitochondrial dysfunction-related oxidative stress in fibroblasts of patients with Leigh syndrome by inhibition of prooxidative p66Shc pathway. Mitochondrion 37:62-79. https://doi.org/ 10.1016/j.mito.2017.07.002

30. Giorgio M, Migliaccio E, Orsini F et al (2005) Electron transfer between cytochrome $\mathrm{c}$ and $\mathrm{p} 66 \mathrm{Shc}$ generates reactive oxygen species that trigger mitochondrial apoptosis. Cell 122(2):221-233. https://doi.org/10.1016/j.cell.2005.05.011

31. De Bock K, Georgiadou M, Schoors S et al (2013) Role of PFKFB3-driven glycolysis in vessel sprouting. Cell 154(3):651663. https://doi.org/10.1016/j.cell.2013.06.037

32. Schoors S, Bruning U, Missiaen R et al (2015) Fatty acid carbon is essential for dNTP synthesis in endothelial cells. Nature 520(7546): 192-197. https://doi.org/10.1038/nature14362

33. Sawada N, Arany Z (2017) Metabolic regulation of angiogenesis in diabetes and aging. Physiology 32(4):290-307. https://doi.org/10. 1152/physiol.00039.2016
34. Heiss EH, Schachner D, Donati M, Grojer CS, Dirsch VM (2016) Increased aerobic glycolysis is important for the motility of activated VSMC and inhibited by indirubin-3'-monoxime. Vasc Pharmacol 83:47-56. https://doi.org/10.1016/j.vph.2016.05.002

35. King GL, Buzney SM, Kahn CR et al (1983) Differential responsiveness to insulin of endothelial and support cells from micro- and macrovessels. J Clin Investig 71(4):974-979. https://doi.org/10.1172/JCI110852

36. Mandarino LJ, Finlayson J, Hassell JR (1994) High glucose downregulates glucose transport activity in retinal capillary pericytes but not endothelial cells. Invest Ophthalmol Vis Sci 35(3):964-972

37. Divakaruni AS, Brand MD (2011) The regulation and physiology of mitochondrial proton leak. Physiology 26(3):192-205. https:// doi.org/10.1152/physiol.00046.2010

38. Cheng J, Nanayakkara G, Shao Y et al (2017) Mitochondrial proton leak plays a critical role in pathogenesis of cardiovascular diseases. Adv Exp Med Biol 982:359-370. https://doi.org/10.1007/978-3319-55330-6_20

39. Pomero F, Allione A, Beltramo E et al (2003) Effects of protein kinase $\mathrm{C}$ inhibition and activation on proliferation and apoptosis of bovine retinal pericytes. Diabetologia 46(3):416-419. https://doi. org/10.1007/s00125-003-1044-5

40. Amadio M, Scapagnini G, Lupo G, Drago F, Govoni S, Pascale A

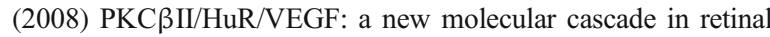
pericytes for the regulation of VEGF gene expression. Pharmacol Res 57(1):60-66. https://doi.org/10.1016/j.phrs.2007.11.006

41. Geraldes P, Hiraoka-Yamamoto J, Matsumoto M et al (2009) Activation of PKC- $\delta$ and SHP-1 by hyperglycemia causes vascular cell apoptosis and diabetic retinopathy. Nat Med 15:1298-1306. https://doi.org/10.1038/nm.2052

42. Mustapha NM, Tarr JM, Kohner EM, Chibber R (2010) NADPH oxidase versus mitochondria-derived ROS in glucose-induced apoptosis of pericytes in early diabetic retinopathy. J Ophthalmol 2010:746978. https://doi.org/10.1155/2010/746978

43. Sena CM, Leandro A, Azul L, Seiça R, Perry G (2018) Vascular oxidative stress: impact and therapeutic approaches. Front Physiol 9:1668. https://doi.org/10.3389/fphys.2018.01668

44. Islam MS, Leissing TM, Chowdhury R, Hopkinson RJ, Schofield CJ (2018) 2-Oxoglutarate-dependent oxygenases. Annu Rev Biochem 87(1):585-620. https://doi.org/10.1146/annurevbiochem-061516-044724

45. Bayliak MM, Shmihel HV, Lylyk MP et al (2015) Alpha-ketoglutarate attenuates toxic effects of sodium nitroprusside and hydrogen peroxide in Drosophila melanogaster. Environ Toxicol Pharmacol 40(2):650 659. https://doi.org/10.1016/j.etap.2015.08.016

46. Murphy MP (2009) How mitochondria produce reactive oxygen species. Biochem J 417(1):1-13. https://doi.org/10.1042/BJ20081386

47. Yao K, Yin Y, Li X et al (2012) Alpha-ketoglutarate inhibits glutamine degradation and enhances protein synthesis in intestinal porcine epithelial cells. Amino Acids 42(6):2491-2500. https:// doi.org/10.1007/s00726-011-1060-6

48. Jiang Q, Liu G, Wang X et al (2017) Mitochondrial pathway is involved in the protective effects of alpha-ketoglutarate on hydrogen peroxide induced damage to intestinal cells. Oncotarget 8(43): 74820-74835. https://doi.org/10.18632/oncotarget.20426

49. Shahid M, Gull N, Yeon A et al (2018) Alpha-oxoglutarate inhibits the proliferation of immortalized normal bladder epithelial cells via an epigenetic switch involving ARID1A. Sci Rep 8(1):4505. https://doi.org/10.1038/s41598-018-22771-2

50. Matsumoto K, Obara N, Ema M et al (2009) Antitumor effects of 2oxoglutarate through inhibition of angiogenesis in a murine tumor model. Cancer Sci 100(9):1639-1647. https://doi.org/10.1111/j. 1349-7006.2009.01249.x

Publisher's note Springer Nature remains neutral with regard to jurisdictional claims in published maps and institutional affiliations. 